\title{
Stable Luminescence of Single Quantum Emitters: Applications in Quantum Optics
}

\author{
A.V.Naumov ${ }^{1,2 a}$, I.Y.Eremchev ${ }^{1}$, A.A.Gorshelev ${ }^{1}$, M.G.Gladush ${ }^{1}$, L.Kador ${ }^{3}$, and J.Köhler ${ }^{3}$ \\ ${ }^{1}$ Institute for Spectroscopy RAS, Fizicheskaya Str. 5, Troitsk, Moscow 142190, Russia \\ ${ }^{2}$ Moscow Pedagogical State University, Malaya Pirogovskaya Str. 29, Moscow 119991, Russia \\ ${ }^{3}$ Bayreuth University and Bayreuth Institute for Macromolecular Researches, 95447, Bayreuth, Germany
}

\begin{abstract}
In our work, we demonstrate the advantages and drawbacks of the methods for generating nonclassical light using single luminescent molecules in solid matrices at cryogenic temperatures when excitation of zero-phonon spectral lines is possible. It is shown that for certain impurity-matrix systems it is possible to guarantee the following: single-photon generation at a rate of up to tens-hundreds $\mathrm{MHz}$, allocation of an extremely narrow generation band (a few MHz), wavelength tuning over a wide range (tens of nanometers), generation of biphoton radiation, high photostability and absence of a stochastic spectral dynamics.
\end{abstract}

Keywords: single-molecule, zero-phonon line, dye center, photoluminescence, single photon source, biphoton source

Beginning with the pioneering papers on the detection of fluorescence of single molecules (SM) [1], it became clear that a single molecule in a solid matrix, luminescenting in the "zero-phonon mode" (i.e., emitting light as a result of phototransitions between the electron-vibrational levels without changing the number of phonons of the matrix) is an ideal candidate for the role of a nonclassical light source a single-photon generator. The subsequent studies (see [2-4] for review) of different environments give a good possibility to identify the main strengths and weaknesses of the potential photon generators based on SM zero-phonon spectral lines (ZPL).

Among the advantages we should note the following:

- Guaranteed single-photon emission (the probability of multiphoton processes is usually by orders of magnitude lower than normal luminescence);

- High generation rate is inversely proportional to the lifetime of the excited state (which for traditional chromophore molecules can reach several $\mathrm{GHz}$ );

- Extremely narrow generation band (homogeneous width of SM ZPL at liquid helium temperatures reaches the values of a few $\mathrm{MHz}$ );

- The possibility of implementing a broadband wavelength tuning by selection of a particular SM within a wide inhomogeneous absorption contour of the impurity medium (given that the ratio of heterogeneous and homogeneous widths in disordered solid media at cryogenic temperatures can reach $10^{5}-10^{6}$, wavelength tuning is possible within the range of up to several hundred $\mathrm{nm}$ );

${ }^{\text {a }}$ Corresponding author: a_v_naumov@mail.ru

This is an Open Access article distributed under the terms of the Creative Commons Attribution License 4.0, which permits unrestricted use, distribution, and reproduction in any medium, provided the original work is properly cited. 
- Strict positioning of SM source in a solid matrix (which greatly facilitates experimental implementation as compared to single atoms lions in traps).

- The possibility to choose the proper dye-matrix system with appropriate photophysical properties (Debye-Waller factor, temperature dependence of properties, spectral range, etc.). [5,6]

At the same time, there are serious obstacles to the creation of single-photon sources based on SM ZPL:

- The presence of spectral diffusion stochastic processes, leading to random walks (reversible and irreversible jump/drift) of ZPL frequency;

- Photochemical and photophysical "burning" of SMs (photobleaching);

- Multiple of "channels" of the SM excited state decay into large numbers of the vibronic levels of the ground state, which, on the one hand increases the effective spectral width of the source, and, on the other hand, restricts the control over the phase of the emitted light;

- The complexity of the experimental realization and operation of the cryogenic source. [7]

In recent papers $[8,9]$ it was discovered that SM spectral diffusion can be significantly suppressed in a number of impurity solid media - frozen solutions of terrylene molecules in the ortho and paradichlorobenzene.

The studies have shown that these systems exhibit unique photophysical and dynamic properties:

- ZPL widths close to the maximum (from few MHz and higher);

- High photostability of chromophore molecules (up to the power density of the exciting laser at few to tens of $\mathrm{MW} / \mathrm{cm}^{2}$ );

- High luminescence intensity of SMs (up to $10^{6}$ photons per second and higher);

- Almost complete absence of the slow spectral dynamics (very low contribution of the tunneling two-level systems to SM spectral dynamics observed at $\mathrm{T}<2-5 \mathrm{~K}$ );

- Large inhomogeneous broadening of the optical spectrum (it allows a controlled wavelength tuning within the range greater than $100 \mathrm{~nm}$ ).

Further studies have provided evidence indicating the possibility of generating correlated photons from emitting pairs of dipole-dipole interacting terrylene molecules (like in [10]) located 1-5 nm apart.

This list of possible techniques to generate in an experiment highly stable narrow ZPL of SM in solid matrices in the absence of spectral diffusion suggests the possibility of their effective use in nanophotonic and quantum information devices as sources of nonclassical light (tunable single and biphoton sources).

The support from Russian Foundation for Basic Research is acknowledged (13-02-01303).

\section{References}

1. M. Orrit, J. Bernard, Phys. Rev. Lett. 65 P.2716 (1990)

2. T. Basché, W.E. Moerner, M. Orrit, U.P. Wild, Single-molecule optical detection, imaging and spectroscopy (Weinheim; Cambridge: VCH, xiv, 250 p., 1997)

3. M. Orrit, W.E. Moerner, High Resolution Single-Molecule Spectroscopy in Condensed Matter, Physics and Chemistry at Low Temperatures (L. Khriachtchev, ed.) (Pan Stanford Publishing, Singapore, p. 381-417, 2011)

4. A.V. Naumov, Physics Uspekhi. 56. P.605-622 (2014)

5. N.L. Naumova, I.A. Vasil'eva, I.S. Osad'ko, A.V. Naumov, J. Lumin. 111, 37 (2005)

6. K.R. Karimullin, A.V. Naumov, J. Lumin. 152, P.15 (2014)

7. A.V. Naumov, I.Yu. Eremchev, A.A. Gorshelev, Eur. Phys. J. D. 68, P. 348 (2014)

8. A.A. Gorshelev, A.V. Naumov, I.Y. Eremchev, Y.G. Vainer, L. Kador, J. Kohler, ChemPhysChem. 1, P.182 (2010)

9. P. Navarro, Y. Tian, M. van Stee, M. Orrit, ChemPhysChem. 15, P.3032 (2014)

10. C. Hettich, C. Schmitt, J. Zitzmann, S. Kuhn, I. Gerhardt, V. Sandoghdar, Science, 298, P.385-389 (2002) 